\title{
A Genetically Engineered STO Feeder System Expressing E-Cadherin and Leukemia Inhibitory Factor for Mouse Pluripotent Stem Cell Culture
}

Masanobu Horie, Akira Ito, Yoshinori Kawabe and Masamichi Kamihira*

Department of Chemical Engineering, Faculty of Engineering, Kyushu University, 744 Motooka, Nishi-ku, Fukuoka 819-0395, Japan

\begin{abstract}
Embryonic stem (ES) cells and induced pluripotent stem (iPS) cells are usually cultured on mouse embryonic fibroblasts (MEFs) isolated from fetuses. MEFs are primary cells and can only be cultured for several passages before senescence. Although the STO mouse stromal cell line has been used as a MEF substitute, performance of a STO feeder layer for ES/iPS cell culture is inferior to a MEF feeder layer. Thus, the development of effective feeder systems may be beneficial for advancing stem cell technology using ES/iPS cells. We established a STO feeder cell line expressing mouse leukemia inhibitory factor (LIF) and mouse E-cadherin (designated as STO/EL cells). ES/iPS cells were cultured on STO/EL feeder cells without LIF addition to the medium, while maintaining the expression of stem cell markers, Oct3/4, Nanog and Rex1. Quantitative evaluation of feeder performance by an alkaline phosphatase-positive colony-forming assay revealed that the colony forming efficiency was comparable to that of the conventional and most reliable culture method using MEFs with LIF addition. STO/EL cells can be used as an efficient feeder system for supporting ES/iPS cell culture in an undifferentiated state. The STO/EL feeder system may contribute toward medical and biological research of ES/iPS cells.
\end{abstract}

Keywords: Embryonic stem cell; Induced pluripotent stem cell; Feeder cell; E-cadherin; LIF; STO

Abbreviations: AP: Alkaline Phosphatase; CM: Conditioned Medium; ES: Embryonic Stem; iPS: induced Pluripotent Stem; LIF: Leukemia Inhibitory Factor; LPA: Lysophosphatidic Acid; MEF: Mouse Embryonic Fibroblasts; ROCK: Rho-associated Coiled-Coil Forming Kinase

\section{Introduction}

Pluripotent cell lines such as ES cells derived from embryos and iPS cells generated from somatic cells have become important tools for medical and biological research [1,2]. These pluripotent cells are characterized by self-renewal and a differentiation potential for all cell types of the adult organism. A robust culture system for maintaining the self-renewal of ES/iPS cells and differentiation potential is crucial for research. Undifferentiated ES/iPS cell culture is usually supported by a feeder cell layer in the presence of anti-differentiation factors such as LIF. MEFs isolated from fetuses are often used as feeder cells for mouse and human ES/iPS cell culture. However, MEFs are primary cells and can only be cultured for several passages before senescence. Although the STO mouse stromal cell line has been used as a substitute for MEFs, the performance as a feeder for ES/iPS cell culture is inferior to MEFs. The detailed mechanism for supporting the undifferentiated state of ES/iPS cells on a MEF feeder layer remains unclear. In addition, STO cells appear to produce few or less growth factors to support ES/iPS cell culture. Thus, we theorized that close contact between STO and ES cells would improve the performance of STO cells as feeders for ES cell culture. E-cadherin, a $\mathrm{Ca}^{2+}$-dependent cell-cell adhesion molecule, plays important roles in intercellular adhesion, colony formation and differentiation of ES cells [3]. Previously, we generated E-cadherinexpressing STO cells that promote cell-cell interactions with ES cells inherently expressing E-cadherin, and demonstrated improved performance comparable to that of a MEF feeder layer [4].

In our E-cadherin gene-engineered feeder system, LIF addition to the culture medium was essential. In the present study, we constructed genetically engineered STO cells co-expressing E-cadherin and LIF to further improve the performance of STO cells as feeders for ES/iPS cell culture.

\section{Materials and Methods}

\section{Cell culture}

Mouse ES cell lines, H-1 (Riken BioResource Center, Tsukuba, Japan) and $129 / \mathrm{Sv}$ (Chemicon, Pittsburgh, PA, USA), and a mouse iPS cell line, iPS-MEF-Ng-20D-17 [2] (Riken BioResource Center) were maintained on mitotically inactivated feeder cells that were treated with mitomycin $\mathrm{C}$ for $2 \mathrm{~h}$. Cells were cultured on $0.1 \%$ gelatin- $(\mathrm{Na}-$ calai Tesque, Kyoto, Japan) coated tissue culture dishes (Greiner Bioone, Frickenhausen, Germany) in ES/iPS medium consisting of Knockout-DMEM ${ }^{\mathrm{TM}}$ (Invitrogen, Carlsbad, CA, USA) supplemented with $4 \mathrm{mM}$ L-glutamine (Wako Pure Chemical Industries, Osaka, Japan), non-essential amino acids (NEAA; Invitrogen), $100 \mu \mathrm{M}$ 2-mercaptoethanol (Millipore, Billerica, MA, USA), $100 \mathrm{U} / \mathrm{ml}$ penicillin G potassium (Wako Pure Chemical Industries), $50 \mu \mathrm{g} / \mathrm{ml}$ streptomycin sulfate (Wako Pure Chemical Industries) and 15\% Knock-out-serumreplacement (Invitrogen). For routine ES/iPS cell culture, $1 \times 10^{3} \mathrm{U} / \mathrm{ml}$ LIF (ESGRO ${ }^{\mathrm{TM}}$; Millipore) was added to the medium. Culture medium was changed every day, and cells were passaged every $2-3 \mathrm{~d}$.

MEFs were isolated from the fetuses of $14 \mathrm{~d}$ pregnant $\mathrm{BALB} / \mathrm{c}$ mice and cultured in Dulbecco's modified Eagle's medium (DMEM; SigmaAldrich, St. Louis, MO, USA) supplemented with $10 \%$ fetal bovine serum (FBS; Biowest, Miami, FL, USA) and $4 \mathrm{mM} \mathrm{L}$-glutamine. STO

*Corresponding author: Masamichi Kamihira, Department of Chemical Engineering, Faculty of Engineering, Kyushu University, 744 Motooka, Nishi-ku, Fukuoka 819-0395, Japan. Tel.: +81-(0)92-802-2743; Fax: +81-(0)92-802-2793; E-mail: kamihira@chem-eng.kyushu-u.ac.jp

Received July 13, 2011; Accepted September 22, 2011; Published Septembe 28, 2011

Citation: Horie M, Ito A, Kawabe Y, Kamihira M (2011) A Genetically Engineered STO Feeder System Expressing E-Cadherin and Leukemia Inhibitory Factor for Mouse Pluripotent Stem Cell Culture. J Bioprocess Biotechniq S3:001 doi:10.4172/2155-9821.S3-001

Copyright: $\odot 2010$ Horie M, et al. This is an open-access article distributed under the terms of the Creative Commons Attribution License, which permits unrestricted use, distribution, and reproduction in any medium, provided the original author and source are credited. 
cells were cultured in DMEM supplemented with $10 \% \mathrm{FBS}, 100 \mathrm{U} / \mathrm{ml}$ penicillin $\mathrm{G}$ potassium, $50 \mathrm{\mu g} / \mathrm{ml}$ streptomycin sulfate and NEAA. Cells were cultured at $37^{\circ} \mathrm{C}$ in a $5 \%(\mathrm{v} / \mathrm{v}) \mathrm{CO} 2$ incubator. Animal experimentation was approved by the Ethics Committee for Animal Experiments of the Faculty of Engineering, Kyushu University (A21-098-1).

\section{Establishment of E-cadherin and LIF expressing STO cells}

The expression plasmid vector for E-cadherin, pcDNA4/E-cadIRES-EGFP [5] was transfected into STO cells using a lipofection reagent (Lipofectamine2000; Invitrogen). Cells were selected in medium containing $1 \mathrm{mg} / \mathrm{ml}$ zeocin (Invitrogen), and stable E-cadherin-expressing clones (designated as STO/E cells) were established by a limiting dilution method [4]. For construction of an expression plasmid vector for LIF, LIF cDNA in pMFGmLIF [6] (Riken BioResource Center) was PCR amplified using the primers, 5'-CCG CTC GAG ACC ATG AAG GTC TTG GCC GCA G-3' and 5'-GGA ATT CCT AGA AGG CCT GGA CCA CCA C-3' to insert XhoI and EcoRI digestion sites (underlined), respectively. PCR was performed using KOD plus DNA polymerase (Toyobo, Osaka, Japan) at an initial denaturation of $94^{\circ} \mathrm{C}$ for 2 min, followed by 30 cycles of amplification at $94^{\circ} \mathrm{C}$ for $15 \mathrm{~s}, 56^{\circ} \mathrm{C}$ for 30 $\mathrm{s}$ and $68^{\circ} \mathrm{C}$ for $37 \mathrm{~s}$. The PCR product was digested with the relevant restriction enzymes and ligated into a XhoI and EcoRI-digested pIRES2 vector from pIRES2-DsRed-Express (Clontech, Palo Alto, CA, USA) to generate pmLIF. STO and STO/E cells were transfected with pmLIF by electroporation using a Neon transfection system (Invitrogen). Cells

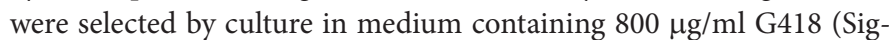
ma-Aldrich). Stable LIF-expressing clones (designated as STO/L cells), as well as E-cadherin and LIF co-expressing clones (designated as STO/ EL cells) were established by a limiting dilution method.

\section{RT-PCR analysis}

After plating ES/iPS cells onto gelatin-coated dishes to remove feeder cells, total RNA was extracted from ES/iPS cells using RNAiso Plus reagent (Takara Bio, Otsu, Japan). RNA was reverse-transcribed into cDNA from $1 \mu \mathrm{g}$ total RNA using a ReverTra Ace First Strand cDNA synthesis kit (Takara Bio). Specific gene sequences were PCR amplified using the primers shown in Table 1.

\section{Western blot analysis}

Cell lysates (50 $\mu$ g protein) of STO, STO/E, STO/L and STO/EL cells were subjected to SDS-PAGE in a $10 \%(\mathrm{w} / \mathrm{v})$ polyacrylamide gel. Proteins were then transferred onto a polyvinylidene difluoride membrane (GE Healthcare, Buckinghamshire, UK). After blocking with a $5 \%(\mathrm{w} / \mathrm{v})$ skim milk solution, membranes were incubated with a rabbit anti-E-cadherin monoclonal antibody (Santa Cruz Biotechnology, Santa Cruz, CA, USA) or rabbit anti-mouse LIF polyclonal antibody (Santa Cruz Biotechnology) for $1 \mathrm{~h}$, and probed with a peroxidase-labeled anti-rabbit antibody (Santa Cruz Biotechnology) for $1 \mathrm{~h}$. Proteins were detected with an ECL detection system (GE Healthcare).

\section{Alkaline phosphatase (AP) staining}

Cells were fixed in $4 \%$ paraformaldehyde for $5 \mathrm{~min}$ at room temperature and treated with a solution containing naphthol AS-MX phosphate (Sigma-Aldrich) as a substrate and Fast Violet B Salt (SigmaAldrich) as a coupler for $20 \mathrm{~min}$ at $37^{\circ} \mathrm{C}$. Cells showing AP activity stained dark brown and were observed with a phase-contrast microscope (Olympus, Tokyo, Japan).

\begin{tabular}{|l|l|}
\hline Target gene (product size) & Primer sequence \\
\hline Actc1 (124 bp) & FW: 5'-CCA GAT CAT GTT TGA GAC CTT CAA-3' \\
RV: 5'-GAA CAT TAT GAG TTA CAC CAT CGC-3' \\
\hline \multirow{3}{*}{ BMP2 (249 bp) } & $\begin{array}{l}\text { FW: 5'-GGG ACC CGC TGT CTT CTA GTG TTG C-3' } \\
\text { RV: 5'-TGA GTG CCT GCG GTA CAG ATC TAG } \\
\text { CA-3' }\end{array}$ \\
\hline a-Fetoprotein (173 bp) & $\begin{array}{l}\text { FW: 5'-TCG TAT TCC AAC AGG AGG-3' } \\
\text { RV: 5'-AGG CTT TTG CTT CAC CAG-3' }\end{array}$ \\
\hline \multirow{5}{*}{ Gata4 (207 bp) } & $\begin{array}{l}\text { FW: 5'-CTG GAG GCG AGA TGG GAC GGG ACA } \\
\text { CTA C-3' } \\
\text { RV: 5'-CCG CAG GCA TTA CAT ACA GGC TCA } \\
\text { CC-3' }\end{array}$ \\
\hline GAPDH (150 bp) & $\begin{array}{l}\text { FW: 5'-CTA CCC CCA ATG TGT CCG TC-3' } \\
\text { RV: 5'-GCT GTT GAA GTC GCA GGA GAC-3' }\end{array}$ \\
\hline Nanog (163 bp) & $\begin{array}{l}\text { FW: 5'-GCG GCT CAC TTC CTT CTG ACT T-3' } \\
\text { RV: 5'-GAC CAG GAA GAC CCA CAC TCA T-3' }\end{array}$ \\
\hline Neurod3/ngn1 (405 bp) & $\begin{array}{l}\text { FW: 5'-CAT CTC TGA TCT CGA CTG C-3' } \\
\text { RV: 5'-CCA GAT GTA GTT GTA GGC G-3' }\end{array}$ \\
\hline \multirow{3}{*}{ Oct3/4 (459 bp) } & $\begin{array}{l}\text { FW: 5'-CTG AGG GCC AGG CAG GAG GAG CAC } \\
\text { GAG-3' } \\
\text { RV: 5'-CTG TAG GGA GGG CTT CGG GCA CTT-3' }\end{array}$ \\
\hline Zfp42/Rex1 (287 bp) & $\begin{array}{l}\text { FW: 5'-ACG AGT GGC AGT TTC TTC TTG GGA-3' } \\
\text { RV: 5'-TAT GAC TCA CTT CCA GGG GGC ACT-3' }\end{array}$ \\
\hline \multirow{2}{*}{ Table 1: } & Primer sequences for RT-PCR analysis \\
\hline
\end{tabular}

\section{ES/iPS cell culture using conditioned medium (CM)}

STO, STO/E, STO/L and STO/EL cells treated with mitomycin C for $2 \mathrm{~h}$ were seeded at $5 \times 10^{5}$ cells/well in 6 well culture plates. After $1 \mathrm{~d}$, medium was replaced with fresh LIF-free ES/iPS medium and cultures were continued for a further $1 \mathrm{~d}$. Culture supernatants were then collected and filtered through a $0.45 \mu \mathrm{m}$ cellulose acetate filter (Advantec, Tokyo, Japan). CM was prepared by mixing each culture supernatant with fresh LIF-free ES/iPS medium at a ratio of 1:1. 129/Sv cells were cultured for $10 \mathrm{~d}$ on a MEF feeder layer in each CM without LIF and then $1 \times 10^{4}$ cells were re-plated onto a fresh MEF feeder layer. After 3 $\mathrm{d}$ culture in ES/iPS medium containing $1 \times 10^{3} \mathrm{U} / \mathrm{ml} \mathrm{LIF}$, AP-positive colonies were counted.

\section{Hanging drop assay}

Embryoid bodies (EBs) were induced using the hanging drop method [7]. After harvesting ES/iPS cells, the cells were re-suspended in LIF-free ES/iPS medium at $7 \times 10^{3}$ cells $/ \mathrm{ml}$. Droplets of the cell suspension $(15 \mu \mathrm{l})$ were placed on the lid of a bacterial grade $100 \mathrm{~mm}$ plastic dish (AsOne, Osaka, Japan). The lid was inverted and placed on the bottom half of a dish filled with phosphate buffered saline (PBS) and then incubated at $37^{\circ} \mathrm{C}$ in a $5 \% \mathrm{CO}_{2}$ incubator. After $2 \mathrm{~d}$, cells were transferred to gelatin-coated dishes and cultured in DMEM supplemented with $15 \% \mathrm{FBS}$, NEAA, $100 \mathrm{U} / \mathrm{ml}$ penicillin G potassium and 50 $\mu \mathrm{g} / \mathrm{ml}$ streptomycin sulfate.

\section{Colony forming assay}

ES/iPS cells were cultured for $10 \mathrm{~d}$ on the various feeder layers in ES/iPS medium with or without LIF and then $1 \times 10^{4}$ cells were replated onto a MEF feeder layer. After $2 \mathrm{~d}$ culture in ES/iPS medium containing $1 \times 10^{3} \mathrm{U} / \mathrm{ml}$ LIF, AP staining was performed as described above. AP-positive colonies were counted using microscope images from five fields of view in three separate wells per sample.

\section{Magnetic force-based culture}

Magnetically-labeled ES/iPS cells and a magnetic force were used to enhance the physical contact between ES/iPS cells and feeder lay- 
ers [4]. Magnetite cationic liposomes (MCLs) were prepared from colloidal magnetite nanoparticles $\left(\mathrm{Fe}_{3} \mathrm{O}_{4}, 10 \mathrm{~nm}\right.$ average particle size; Toda Kogyo, Hiroshima, Japan) and a lipid mixture consisting of $\mathrm{N}$-( $\alpha$-trimethylammonioacetyl)-didodecyl-D-glutamate chloride, dilaur+oylphosphatidylcholine and dioleoylphosphatidyl-ethanolamine at a molar ratio of 1:2:2 as described elsewhere [8]. For magnetic labeling of ES/iPS cells, MCLs were added to ES/iPS cell cultures at a net magnetite concentration of $100 \mathrm{pg} / \mathrm{cell}$. In our previous study, ES cells rapidly took up MCLs and reached $13 \mathrm{pg} /$ cell at $2 \mathrm{~h}$ after MCL addition, and $95 \%$ of cells were magnetically captured, suggesting that almost all cells were labeled with MCLs and the uptake amount was sufficient for magnetic cell attraction [9]. After a $2 \mathrm{~h}$ incubation, $1 \times$ $10^{5} \mathrm{ES} / \mathrm{iPS}$ cells/well were seeded onto feeder layers in 24 well plates. A cylindrical neodymium magnet $(30 \mathrm{~mm}$ diameter; $0.4 \mathrm{~T}$ magnetic induction) was then placed under the wells to apply a vertical magnetic force. Cells were cultured for $7 \mathrm{~d}$, including two passages, under the applied magnetic force. Then, $1 \times 10^{4}$ cells/well were re-plated onto MEFs in 24 well plates. After $3 \mathrm{~d}$ culture, AP-positive colonies were counted.

\section{ROCK inhibitor and Rho activator treatments}

For ROCK inhibitor and Rho activator treatments, Y-27632 (Wako Pure Chemical Industries) or lysophosphatidic acid (LPA; Enzo Life Science, Farmingdale, NY, USA) were respectively added at $10 \mu \mathrm{M}$ to ES/iPS medium. ES/iPS cells were then plated onto feeder layers in ES/ iPS medium containing Y-27632 or LPA. Cells were cultured for $10 \mathrm{~d}, 1$ $\times 10^{4}$ cells/well were then re-plated onto MEFs in 24 well plates. After 3 $\mathrm{d}$ culture, AP-positive colonies were counted.

\section{Statistical analysis}

Data were expressed as the means \pm standard deviation (SD). Statistical comparisons were evaluated using a one-way analysis of variance (ANOVA), and $P<0.05$ was considered significant.

\section{Results}

\section{Establishment of genetically engineered STO cells expressing E-cadherin and LIF}

We previously established E-cadherin-expressing STO cells (STO/E cells) [4]. STO/E feeder layers exhibited excellent performance for supporting undifferentiated culture of mouse ES cells comparable to that of MEF feeder layers in the presence of LIF [4] Figure 1A. However, the effectiveness of STO/E feeder layers was greatly reduced without LIF Figure 1A. In the present study, the LIF gene was introduced into STO and STO/E cells. Transgene expression was analyzed by RT-PCR Figure $1 \mathrm{~B}$, and protein level expression by western blot analysis Figure 1C. Genetically engineered STO cells expressed elevated levels of Ecadherin (STO/E cells), LIF (STO/L cells) and both E-cadherin and LIF (STO/EL cells) compared with those of MEFs and parental STO cells. E-cadherin expression was almost identical between STO/E and STO/ EL cells. From the image analysis of RT-PCR Figure 1B, LIF expression of STO/L cells was 1.7-fold higher than that of STO/EL cells. Western blot analysis Figure 1C revealed that LIF protein level in STO/L cell lysate was 1.2-fold higher than that of STO/EL cells. To evaluate the activity of LIF expressed by genetically modified cells, ES cells were cultured on MEF feeder layers in CM prepared from STO/L and STO/ EL cell culture supernatants. AP-positive ES cell colonies were counted after $10 \mathrm{~d}$ culture (3 passages) Figure 1D. As compared with conventional ES cell cultures using ES/iPS medium containing $1 \times 10^{3} \mathrm{U} / \mathrm{ml}$ LIF, the number of AP-positive colonies decreased in CM from STO and STO/E cells. However, the efficiency of the medium was improved using CM from STO/L and STO/EL cells. For ES/iPS culture in STO/L $\mathrm{CM}$, the number of AP-positive colonies was comparable to that of ES/ iPS culture in medium containing $1 \times 10^{3} \mathrm{U} / \mathrm{ml} \mathrm{LIF}$, while AP-positive colonies in STO/EL CM was slightly lower compared with that of using STO/L CM. These results indicated that LIF expression in STO/EL cells was lower compared with that of STO/L cells, and was consistent with RT-PCR results Figure 1B.

\section{Stem cell marker expression and pluripotency analyses of ES/ iPS cells cultured on STO/EL feeders}

Two mouse ES cell lines (H-1 and 129/Sv cells) and a mouse iPS

A

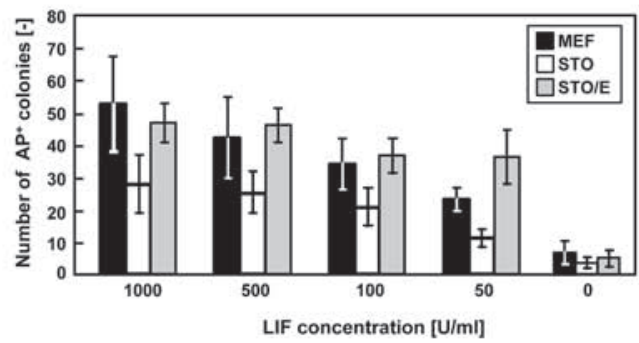

B

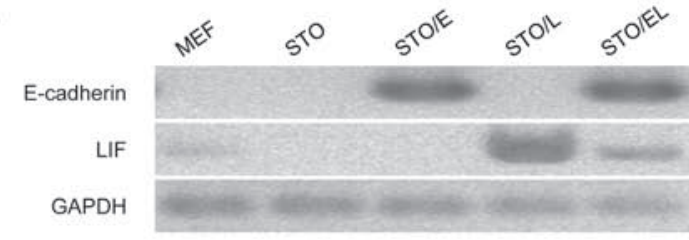

C

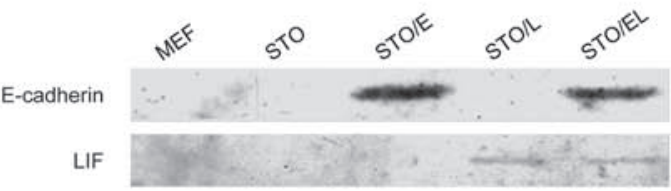

D

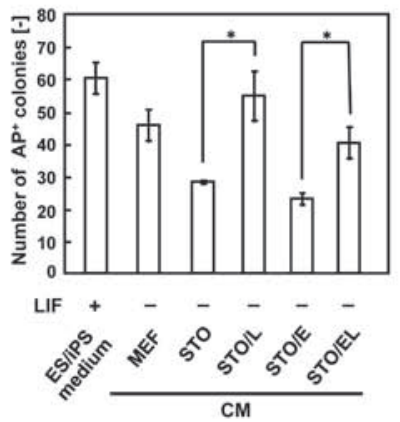

Figure 1: STO/EL cell establishment.

(A) Effect of LIF concentration on maintaining ES cell cultures in an undifferentiated state. 129/Sv cells were cultured in various LIF concentrations $(0-1,000 \mathrm{U} / \mathrm{ml}$ ) on MEF (black column), STO (white column) and STO/E (gray column) feeder layers. ES cells were re-plated onto a MEF feeder layer and cultured in medium containing $1 \times 10^{3} \mathrm{U} / \mathrm{ml}$ LIF for $2 \mathrm{~d}$. Then, AP-positive ES colonies were counted.

(B) RT-PCR analysis of E-cadherin and LIF expression in STO/EL cells. (C) Western blot analysis of E-cadherin and LIF expression in STO/EL cells. (D) Effect of CM on the number of AP-positive ES cell colonies. 129/Sv cells were cultured on a MEF feeder layer in CM prepared from the various feeder layers. The control experiment was performed by culturing 129/Sv cells on a $\mathrm{MEF}$ feeder layer in ES/iPS medium containing $1 \times 10^{3} \mathrm{U} / \mathrm{ml}$ LIF. Experiments were performed in triplicate, and data are the means $\pm \mathrm{SD},{ }^{\star} P<0.05$. 
Citation: Horie M, Ito A, Kawabe Y, Kamihira M (2011) A Genetically Engineered STO Feeder System Expressing E-Cadherin and Leukemia Inhibitory Factor for Mouse Pluripotent Stem Cell Culture. J Bioprocess Biotechniq S3:001 doi:10.4172/2155-9821.S3-001

Page 4 of 7

cell line (iPS-MEF-Ng-20D-17) were used to investigate whether STO/ EL cells could support the self-renewal of pluripotent stem cells. ES and iPS cells cultured on MEF, STO, STO/E and STO/L feeder layers in LIF-free medium changed morphology and a decrease in the number of AP-positive cells was observed Figure 2A. iPS cells maintained a relatively undifferentiated state irrespective of which cells were used as feeder layer. However, ES and iPS cells cultured on a STO/EL feeder layer exhibited a typical ES/iPS cell morphology with tightly packed cell colonies and smooth borders Figure 2A. Cell morphology and the number of AP-positive cells were very similar to those of cells cultured on a MEF feeder layer in LIF-containing medium. To further investigate the undifferentiated state of ES/iPS cells cultured on each feeder layer for $10 \mathrm{~d}$ ( 3 passages), stem cell marker expression was analyzed by RT-PCR Figure 2B. The expression levels of Oct3/4, Zfp42/Rex1 and Nanog were detected in ES/ iPS cells cultured on a STO/EL feeder layer. The stem cell marker expression levels were almost identical to those of cells cultured on a MEF feeder layer in LIF-containing medium as the control. However, the expression of stem cell marker genes were low or

A
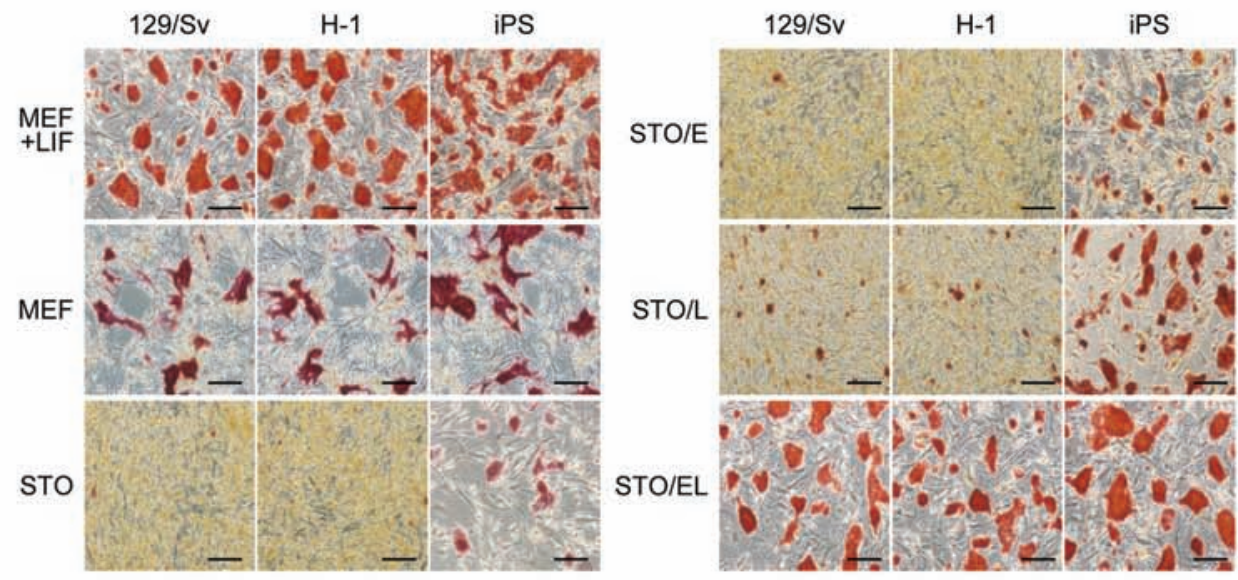

B
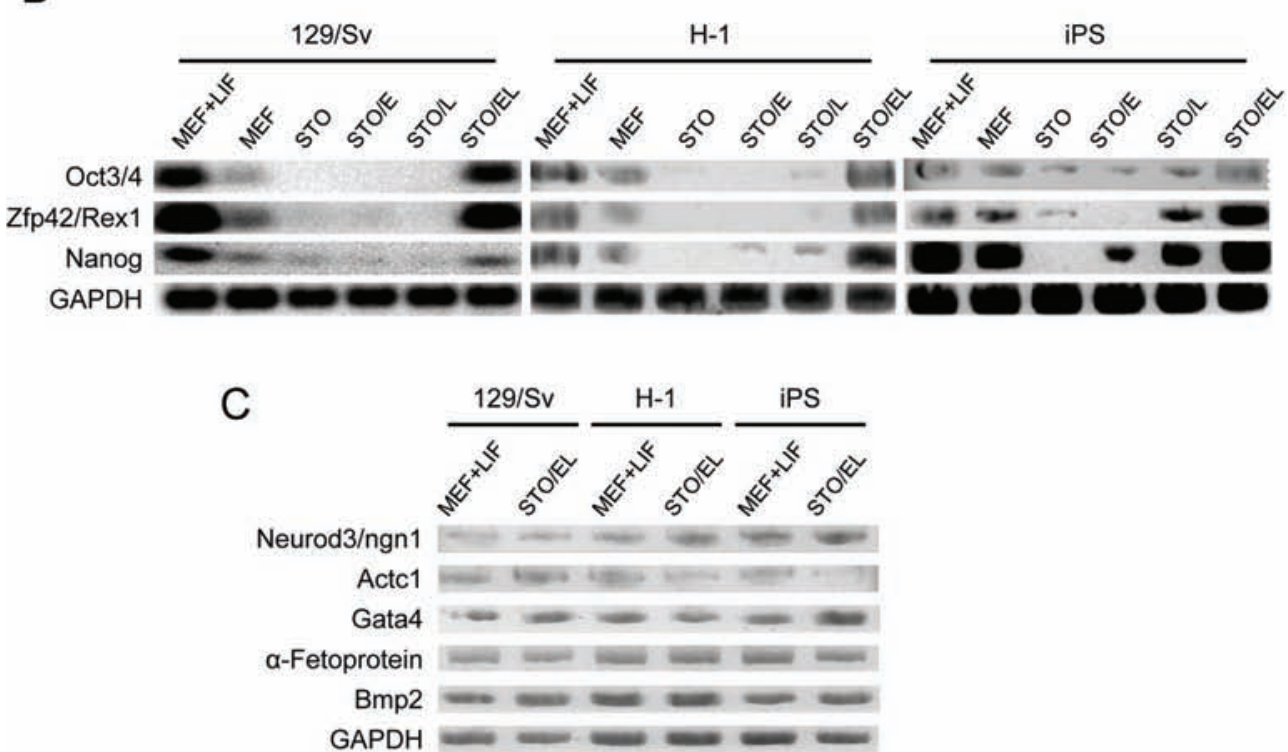

Figure 2: Expression of stem cell marker and pluripotency genes.

(A) AP staining of ES/iPS cells cultured on MEF, STO, STO/E, STO/L and STO/EL feeder layers in medium with (MEF+LIF) or without (MEF, STO, STO/E, STO/L and STO/EL) LIF. Scale bars: $500 \mu \mathrm{m}$.

(B) RT-PCR analysis of genetic markers of the undifferentiated state. ES/iPS cells were cultured on the various feeder layers in LIF-free medium (MEF, STO, STO/E, $\mathrm{STO} / \mathrm{L}$ and STO/EL). The control experiment (MEF+LIF) was performed by culturing ES/iPS cells on a MEF feeder layer in medium containing $1 \times 10^{3} \mathrm{U} / \mathrm{mI}$ LIF.

(C) RT-PCR analysis of marker genes related to formation of the three germ layers. ES/iPS cells were cultured on a STO/EL feeder layer for $10 \mathrm{~d}$ in LIF-free medium. The control experiment (MEF+LIF) was performed by culturing ES/iPS cells on a MEF feeder layer in medium containing $1 \times 10^{3}$ U/ml LIF. ES/iPS cells were then cultured in suspension with differentiation medium to form EBs. After $11 \mathrm{~d}$, the expression of marker genes was analyzed by RT-PCR. 
absent in cells cultured on MEF, STO, STO/E or STO/L feeder layers in LIF-free medium.

The pluripotency of ES/iPS cells cultured on STO/EL feeders in LIF-free medium was investigated by analyzing differentiation capability via EB formation. For EB formation, ES/iPS cells cultured on STO/ EL feeders in LIF-free medium and MEF feeders in LIF-containing medium, as the control, were transferred to suspension culture in differentiation medium. Expression of marker genes related to the formation of the three germ layers was analyzed by RT-PCR Figure 2C. Cells in EBs expressed the marker genes of all three germ layers (Neurod3/ngn1, Actc1, Gata4, $\alpha$-fetoprotein and BMP-2), indicating that in vitro pluri- potency was maintained in ES/iPS cells cultured on STO/EL feeders in LIF-free medium. Moreover, ES/iPS cells cultured for $10 \mathrm{~d}$ on STO/EL feeders in LIF-free medium, which were transplanted into the femurs of SCID mice developed into teratomas at the injection sites (data not shown). These results indicate that STO/EL cells as feeders support the undifferentiated state and pluripotency of ES/iPS cells.

\section{Colony forming assay of ES/iPS cells cultured on STO/EL feeders}

For quantitative evaluation of feeder performance, an AP-positive colony-forming assay was performed on ES/iPS cells after $10 \mathrm{~d}$ culture on the various feeder layers. As shown in Figure 3A, ES/iPS cells cul-
A

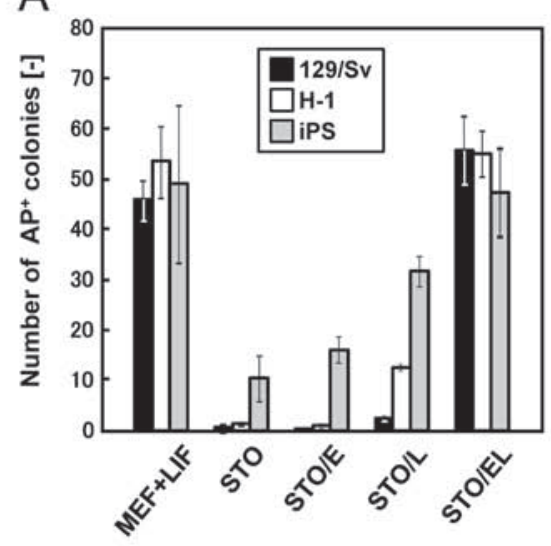

C

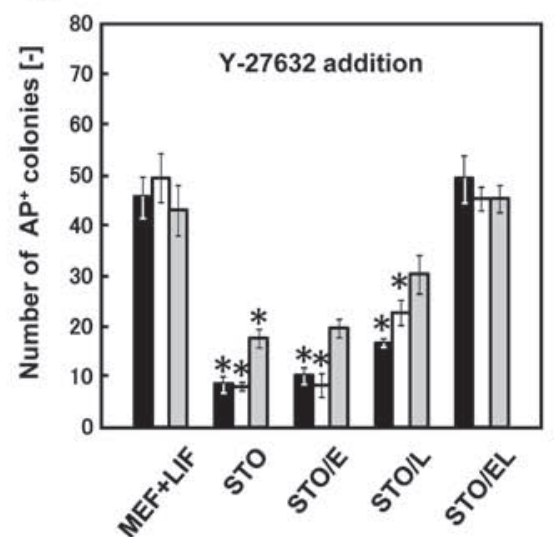

B

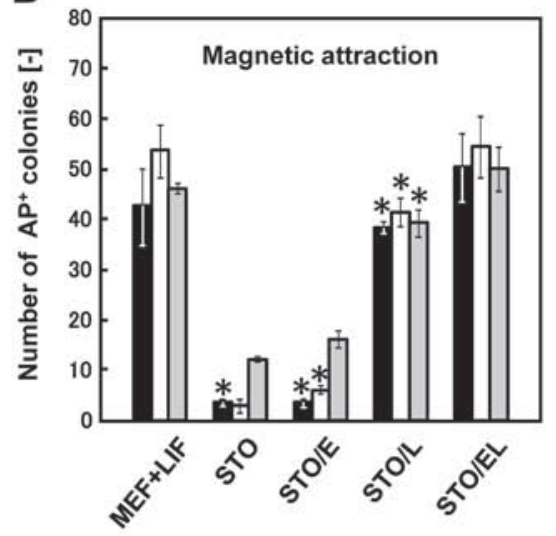

D

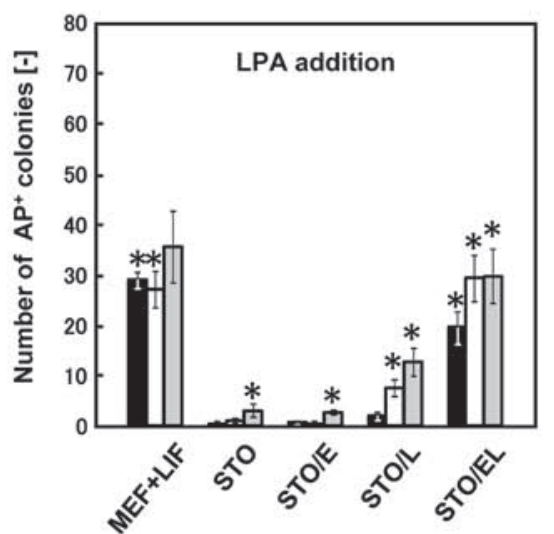

Figure 3: Quantitative analysis of the maintenance of ES/iPS cells in an undifferentiated state by an AP-positive colony forming assay.

(A) ES/iPS cells were cultured on the various feeder layers for $10 \mathrm{~d}$ in LIF-free medium. Then, ES/iPS cells were re-plated onto a MEF feeder layer and cultured in medium containing $1 \times 10^{3} \mathrm{U} / \mathrm{ml}$ LIF for $2 \mathrm{~d}$. The control experiment (MEF+LIF) was performed by culturing ES/iPS cells on a MEF feeder layer in medium containing $1 \times 10^{3} \mathrm{U} / \mathrm{ml}$ LIF.

(B) Effect of the physical interaction of ES/iPS cells with feeder cells using magnetic culture. Magnetically-labeled ES/iPS cells were cultured on the various feeder layers in LIF-free medium under an applied magnetic force for $10 \mathrm{~d}$. The control experiment (MEF+LIF) was performed by culturing magnetically-labeled ES/iPS cells on a MEF feeder layer in medium containing $1 \times 10^{3} \mathrm{U} / \mathrm{ml}$ LIF under an applied magnetic force for $10 \mathrm{~d}$. ES/iPS cells were then re-plated onto a MEF feeder layer and cultured in medium containing $1 \times 10^{3} \mathrm{U} / \mathrm{ml}$ LIF for $2 \mathrm{~d}$. ${ }^{*} P<0.05$ vs the respective data shown in Figure $3 \mathrm{~A}$.

(C) Effect of a ROCK inhibitor on ES/iPS cell culture on the various feeder layers. ES/iPS cells were cultured on the various feeder layers in LIF-free medium containing a ROCK inhibitor (Y-27632) for $10 \mathrm{~d}$. ES/iPS cells were then re-plated onto a MEF feeder layer and cultured in medium containing $1 \times 10^{3} \mathrm{U} / \mathrm{ml} \mathrm{LIF}$ for $2 \mathrm{~d}$. The control experiment (MEF+LIF) was performed by culturing ES/iPS cells on a MEF feeder layer in medium containing $1 \times 10^{3} \mathrm{U} / \mathrm{ml} \mathrm{LIF}$ and $\mathrm{Y}-27632$ for $10 \mathrm{~d}$. ${ }^{\star} P<0.05 \mathrm{vs}$ the respective data shown in Figure 3A.

(D) Effect of a Rho activator on ES/iPS cells cultured on the various feeder layers. ES/iPS cells were cultured on the various feeder layers in LIF-free medium containing a Rho activator (LPA) for $10 \mathrm{~d}$. ES/iPS cells were then re-plated onto a MEF feeder layer and cultured in medium containing $1 \times 10^{3} \mathrm{U} / \mathrm{ml}$ LIF for $2 \mathrm{~d}$. The control experiment (MEF+LIF) was performed by culturing ES/iPS cells on a MEF feeder layer in medium containing $1 \times 10^{3} \mathrm{U} / \mathrm{ml} \mathrm{LIF}$ and LPA for $10 \mathrm{~d}$. *P<0.05 vs the respective data shown in Figure 3A. Experiments were performed in triplicate, and data are the means \pm SD. Black column, 129/Sv; white column, H-1; gray column, iPS-MEFNg-20D-17. 
tured on a STO/EL feeder layer in LIF-free medium formed a higher number of AP-positive colonies compared with those of STO, STO/E or STO/L feeder layers, and the AP level was comparable to that of a MEF feeder in LIF-containing medium.

To enhance the physical interaction between ES/iPS and feeder cells, magnetically-labeled ES/iPS cells were attracted to the feeder cells by magnetic force Figure 3B. The number of AP-positive ES/iPS cell colonies that were magnetically attracted to the MEF feeder layer in the presence of LIF was almost identical to that of cultures without magnetic attraction Figure 3A. Similarly, the number of AP-positive ES/iPS cell colonies cultured on a STO/EL feeder layer did not change in response to magnetic attraction. The ability to support undifferentiated culture of ES cells was slightly improved on STO/E and STO feeder layers in the absence of LIF, but was still relatively low. However, the performance of STO/L feeders was greatly improved by the magnetic attraction.

To investigate whether E-cadherin-mediated signal transduction was involved in maintaining ES/iPS cells in an undifferentiated state via modulation of the Rho-ROCK cascade, an inhibitor and activator of the Rho-ROCK signaling pathway were added to the medium. ES cell culture on STO, STO/E or STO/L feeder layers in LIF-free medium containing the ROCK inhibitor, Y-27632 resulted in significantly increased AP-positive colonies for both cell lines Figure 3C, compared with that of culture without Y-27632 Figure 3A. ROCK inhibitor treatment did not significantly affect iPS cells. However, addition of the Rho and Erk1/2 activator, LPA to the medium resulted in decreased AP-positive colonies for all cell lines and feeder conditions Figure 3D.

\section{Discussion}

Undifferentiated culture of pluripotent stem cells is supported by a complex network of soluble and membrane-bound cytokines, as well as the extracellular matrix provided by stromal feeder cells. The most reliable protocols require freshly isolated MEFs as feeders for the maintenance of ES/iPS cells. However, MEFs exhaust their stem cell supportive properties and undergo senescence after several passages. There have been numerous reports on substitutes for MEFs, such as mouse mesenchymal cell lines STO [10] and 3T3 [4], human placenta cells [11], human amniotic epithelial cells [12] and the mouse testicular stromal cell line JK1 [13]. These feeder cells produce growth factors including LIF, activin A, transforming growth factor $\beta$ (TGF- $\beta$ ), basic fibroblast growth factor (bFGF), Wnts and bone morphogenetic protein-4 (BMP4) $[14,15]$, which are important for the self-renewal of ES/iPS cells. In an analysis of conditioned medium from MEF cultures, 85 proteins were identified and classified into categories such as differentiation and growth factors, and extracellular matrix and remodeling [16]. Importantly, the pluripotency of mouse ES cells is dependent on intracellular signaling including phosphorylation by the Janus family of tyrosine kinases (JAK) via LIF and the LIF receptor, which leads to activation of the signal transducer protein STAT3 [17]. The combination of IL-6 and soluble IL-6 receptor also interacts with and activates a homodimer of gp130, and the gp130-mediated signaling pathway maintains mouse ES cells without involvement of the LIF receptor $[18,19]$. STAT3 activation sufficiently maintains the undifferentiated state of mouse ES cells, and is enhanced by inhibition of the mitogen-activated protein (MAP) kinase (Erk1/2) pathway [17,20]. Human ES cells also express LIF, IL-6 and gp130 receptors, but STAT3 activation is not essential for maintenance of human ES cells in an undifferentiated state. Thus, it has been considered that human ES cells are distinct from mouse ES cells. Recently, Hanna et al. reported that LIF-dependent "naïve" human ES cells, similar to mouse ES cells, could be derived from conventional human ES cells by ectopic induction of Oct4, Klf4 and Klf2 combined with LIF and inhibitors of the glycogen synthase kinase $3 b$ (GSK3b) and Erk1/2 pathways [21]. Therefore, LIF/STAT3 signals may be required to maintain a naïve state of pluripotency even in human ES cells.

In the present study, we developed a STO/EL feeder system to activate JAK/STAT signaling in ES/iPS cells to maintain pluripotency via LIF produced by the feeder cells in LIF-free medium. We demonstrated that the STO/EL feeder layer maintains the pluripotency of ES/iPS cells in LIF-free medium. LIF-expressing STO cells are available from the ECACC cell bank (SNL; EC07032801) [22], and the cells have been used as feeders for ES/iPS cell culture [2,23]. In the present study, the STO/L feeder layer did not sufficiently support undifferentiated culture of ES/iPS cells Figure 3A in LIF-free medium, albeit STO/L cells produced sufficient LIF and the expression level was higher compared with that of STO/EL cells Figure 1C. These results suggest that E-cadherin expression plays a pivotal role in the performance of STO cells as feeders. We hypothesized two possibilities regarding the improved performance of the STO/EL feeder layer; 1) enhanced physical contact between STO and ES/iPS cells facilitates LIF signaling, and/or 2) direct signal transduction via E-cadherin modulation of the Rho-ROCK cascade. The number of AP-positive colonies significantly increased by culturing magnetically-labeled ES/iPS cells that were attracted to STO/L feeder cells by magnetic force Figure 3B. This observation suggests that close physical contact with ES/iPS cells is important for STO feeder layers to perform efficiently. Notably, the feeder performance of STO and STO/E cells in LIF-free ES cell cultures also slightly improved in magnetic culture. Although these results suggest that STO cells produce anti-differentiation factors other than LIF, the production of LIF by feeder cells or medium supplementation is essential to maintain ES/ iPS cell cultures in an undifferentiated state.

Thus far, several signaling pathways other than the JAK/STAT3 pathway have been identified as regulators of self-renewal in ES/iPS cells. Harb et al. reported that the Rho-ROCK-myosin signaling axis determines the cell-cell integrity of self-renewing ES cells [24]. A ROCK inhibitor blocks apoptosis and supports human ES cell proliferation without affecting pluripotency following dissociation into single cells [25]. In this study, ES/iPS cells cultured on each feeder layer with the ROCK inhibitor, Y-27632 or the Rho activator, LPA (also acts as an Erk1/2 activator), resulted in significantly increased Figure 3C or decreased Figure 3D AP-positive colonies. These results indicate that the Rho-ROCK signaling pathway regulates the undifferentiated state of ES/iPS cells in the presence and absence of LIF. Nevertheless, it is difficult to specify the E-cadherin signal from E-cadherin-expressing feeder cells to ES/iPS cells, because ES/iPS cells also express E-cadherin. At least, E-cadherin expression in feeder cells did not affect the RhoROCK signaling pathway in ES/iPS cells, although cell-cell interactions were enhanced between STO and ES/iPS cells. Thus, the Rho-ROCK signaling pathway does not seem to be the dominant mechanism for supporting the undifferentiated state of ES/iPS cells using STO/EL feeder. Taken together, our results suggest that STO/EL cells support undifferentiated culture of ES/iPS cells in LIF-free medium due to enhanced LIF/STAT3 signaling in response to LIF expression via forced physical contact by E-cadherin expression.

Interestingly, the effectiveness of feeder layers for supporting undifferentiated culture of ES/iPS cells in LIF-free medium varies among the three cell lines Figure 3A. iPS cells cultured on STO, STO/E and STO/L feeder layers formed a higher number of AP-positive colonies compared with those of the other two ES cell lines. The gene expression profiles of ES/iPS cells vary among cell lines due to variations in 
Citation: Horie M, Ito A, Kawabe Y, Kamihira M (2011) A Genetically Engineered STO Feeder System Expressing E-Cadherin and Leukemia Inhibitory Factor for Mouse Pluripotent Stem Cell Culture. J Bioprocess Biotechniq S3:001 doi:10.4172/2155-9821.S3-001

establishment procedures and culture history [26, 27], which may affect compatibility with various feeder layers. A MEF feeder layer is the most robust system for maintaining the undifferentiated state of ES/iPS cell cultures in the presence of LIF. In this study, the STO/EL feeder system even in LIF-free medium is compatible with the ES/iPS cell lines used for experimentation, and comparable to that of a MEF feeder system in the presence of LIF.

In conclusion, we demonstrate that E-cadherin and LIF gene transfer into STO cells improves the performance of a STO feeder layer for ES/iPS cell culture in LIF-free medium. The forced cell-cell interaction via E-cadherin between ES/iPS and feeder cells enhanced the cytokine signal through a paracrine mechanism. MEF isolation is a laborious and time-consuming process requiring the sacrifice of mice. Feederfree culture systems for ES cell culture have been reported, but may cause chromosomal instability in ES cells $[28,29]$. Thus, although the long-term stability of ES/iPS cells using a STO/EL feeder layer and application to other pluripotent cell lines including human ES/iPS cells should be examined, the STO/EL feeder system may contribute toward medical and biological research using ES/iPS cells.

\section{References}

1. Passier R, van Laake LW, Mummery CL (2008) Stem-cell-based therapy and lessons from the heart. Nature 453: 322-329.

2. Okita K, Ichisaka T, Yamanaka S (2007) Generation of germline-competent induced pluripotent stem cells. Nature 448: 313-317.

3. Larue L, Antos C, Butz S, Huber O, Delmas V, et al. (1996) A role for cadherins in tissue formation. Development 122: 3185-3194.

4. Horie M, Ito A, Kiyohara T, Kawabe Y, Kamihira M (2010) E-cadherin geneengineered feeder systems for supporting undifferentiated growth of mouse embryonic stem cells. J Biosci Bioeng 110: 582-587.

5. Ito A, Kiyohara T, Kawabe Y, Ijima H, Kamihira M (2008) Enhancement of cell function through heterotypic cell-cell interactions using E-cadherin-expressing NIH3T3 cells. J Biosci Bioeng 105: 679-682.

6. Nakamura H, Kimura T, Ogita K, Koyama S, Tsujie T, et al. (2004) Alteration of the timing of implantation by in vivo gene transfer: delay of implantation by suppression of nuclear factor kappaB activity and partial rescue by leukemia inhibitory factor. Biochem Biophys Res Commun 321: 886-892.

7. Dang SM, Kyba M, Perlingeiro R, Daley GQ, Zandstra PW (2002) Efficiency of embryoid body formation and hematopoietic development from embryonic stem cells in different culture systems. Biotechnol Bioeng 78: 442-453.

8. Shinkai M, Yanase M, Honda H, Wakabayashi T, Yoshida J, et al. (1996) Intracellular hyperthermia for cancer using magnetite cationic liposomes: in vitro study. Jpn. J Cancer Res 87: 1179-1183.

9. Horie M, Ito A, Maki T, Kawabe Y, Kamihira M (2011) Magnetic separation of cells from developing embryoid bodies using magnetite cationic liposomes. J Biosci Bioeng 112: 184-187.

10. Evans MJ, Kaufman MH (1981) Establishment in culture of pluripotential cells from mouse embryos. Nature 292: 154-156.

11. Park Y, Lee SJ, Choi IY, Lee SR, Sung HJ, et al. (2010) The efficacy of human placenta as a source of the universal feeder in human and mouse pluripotent stem cell culture. Cell Reprogram 12: 315-328.

12. Liu T, Cheng W, Liu T, Guo L, Huang Q, et al. (2010) Human amniotic epithelia cell feeder layers maintain mouse embryonic stem cell pluripotency via epigenetic regulation of the c-Myc promoter. Acta Biochim Biophys Sin (Shanghai) 42: 109-115.

13. Kim J, Seandel M, Falciatori I, Wen D, Rafii S (2008) CD34+ testicular stromal cells support long-term expansion of embryonic and adult stem and progenitor cells. Stem Cells 26: 2516-2522.

14. Eiselleova L, Peterkova I, Neradil J, Slaninova I, Hampl A, et al. (2008) Comparative study of mouse and human feeder cells for human embryonic stem cells. Int J Dev Biol 52: 353-363.
15. Lim JW, Bodnar A (2002) Proteome analysis of conditioned medium from mouse embryonic fibroblast feeder layers which support the growth of human embryonic stem cells. Proteomics 2: 1187-1203.

16. Prowse AB, McQuade LR, Bryant KJ, Marcal H, Gray PP (2007) Identification of potential pluripotency determinants for human embryonic stem cells following proteomic analysis of human and mouse fibroblast conditioned media. $J$ Proteome Res 6: 3796-3807.

17. Smith AG (2001) Embryo-derived stem cells: of mice and men. Annu Rev Cell Dev Biol 17: 435-462.

18. Nichols J, Chambers I, Smith A (1994) Derivation of germline competent embryonic stem cells with a combination of interleukin- 6 and soluble interleukin- 6 receptor. Exp Cell Res 215: 237-239.

19. Yoshida K, Chambers I, Nichols J, Smith A, Saito M, et al. (1994) Maintenance of the pluripotential phenotype of embryonic stem cells though direct activation of gp130 signalling pathways. Mech Dev 45: 163-171.

20. Buehr M, Meek S, Blair K, Yang J, Ure J, et al. (2008) Capture of authentic embryonic stem cells from rat blastocysts. Cell 135: 1287-1298.

21. Hanna J, Cheng AW, Saha K, Kim J, Lengner CJ, et al. (2010) Human embry onic stem cells with biological and epigenetic characteristics similar to those of mouse ESCs. Proc Natl Acad Sci U S A 107: 9222-9227.

22. McMahon AP, Bradley A (1990) The Wnt-1 (int-1) proto-oncogene is required for development of a large region of the mouse brain. Cell 62: 1073-1085.

23. Nagy A, Rossant J, Nagy R, Abramow-Newerly W, Roder JC (1993) Derivation of completely cell culture-derived mice from early-passage embryonic stem cells. Proc Natl Acad Sci U S A 90: 8424-8428.

24. Harb N, Archer TK, Sato N (2008) The Rho-Rock-Myosin signaling axis determines cell-cell integrity of self-renewing pluripotent stem cells. PLoS One 3: e3001.

25. Watanabe K, Ueno M, Kamiya D, Nishiyama A, Matsumura M, et al. (2007) A ROCK inhibitor permits survival of dissociated human embryonic stem cells. Nat Biotechnol 25: 681-686.

26. Chin MH, Pellegrini M, Plath K, Lowry WE (2010) Molecular analyses of human induced pluripotent stem cells and embryonic stem cells. Cell Stem Cell 7: 263-269.

27. Boué S, Paramonov I, Barrero MJ, Izpisúa Belmonte JC (2010) Analysis of human and mouse reprogramming of somatic cells to induced pluripotent stem cells. What is in the plate? PLoS One 5: e12664

28. Draper JS, Smith K, Gokhale P, Moore HD, Maltby E, et al. (2004) Recurren gain chromosomes $17 q$ and 12 in cultured human embryonic stem cells. Nat Biotechnol 22: 53-54.

29. Catalina P, Montes R, Ligero G, Sanchez L, de la Cueva T, et al. (2008) Human ESCs predisposition to karyotypic instability: Is a matter of culture adaptation or differential vulnerability among hESC lines due to inherent properties?. Mo Cancer 7: 76 . 